\title{
Thermal radiation effect on an unsteady MHD natural convection Couette flow with Heat and mass transfer
}

\author{
K. Rama Rao* M V Ramana Murthy and S. Hari singh Naik**
}

*Department of Mathematics, Chaithanya Bharathi Institute of Technology (C. B. I. T), Gandipet, Hyderabad, 500075, Andhra Pradesh, India.

** Department of Mathematics and Computer Science.Univ. Science college, Osmania University, Hyderabad $-7$

\section{Abstract:}

${ }^{1}$ Corresponding email address: krrao86@gmail.com; mv.rm50@gmail.com

The numerical solution of natural convection in unsteady hydromagneticCouette flow of a viscous incompressible electrically conducting fluid between two vertical parallel plates in the presence of thermal radiation is obtained here. The fluid is considered to be a gray, absorbing - emitting but non - scattering medium and the Rosseland approximation is used to describe the radiative heat flux in the energy equation. The dimensionless governing coupled, non - linear boundary layer partial differential equations are solved by an efficient, accurate, and extensively validated and unconditionally stable finite difference scheme of the Crank - Nicolson method. Computations are performed for a wide range of the governing flow parameters, viz., the thermal Grashof number, SolutalGrashof number, Magnetic field parameter (Hartmann number), Prandtl number, Thermal radiation parameter and Schmidt number. The effects of these flow parameters on the velocity and temperatureare shown graphically. Finally, the effects of various parameters on the on the skin - friction coefficient and Rate of heat and mass transferat the wall are prepared with various values of the parameters. These findings are in quantitative agreement with earlier reported studies.

Keywords: Heat and Mass transfer; Natural convection; MHD; Couette flow; Thermal radiation; Finite differencemethod.

\section{Council for Innovative Research}

Peer Review Research Publishing System

Journal: INTERNATIONAL JOURNAL OF COMPUTERS \& TECHNOLOGY

Vol 12, No. 8

editor@cirworld.com

www.cirworld.com, www.ijctonline.com 


\section{Nomenclature:}

$h \quad$ Distance between two parallel plates

$H_{o} \quad$ Magnetic field component along $y^{\prime}-$ axis

$C_{p} \quad$ Specific heat at constant pressure

Gr Grashof number

Gc Modified Grashof number

$g \quad$ Acceleration of gravity

$M \quad$ Hartmann number

Pr Prandtl number

Sc Schmidt number

$R \quad$ Thermal radiation parameter

F Accelerating parameter

D Chemical molecular diffusivity

$T^{\prime} \quad$ Temperature of fluid near the plate

$T_{w}^{\prime} \quad$ Temperature of the fluid far away of the fluid from the plate

$T_{h}^{\prime} \quad$ Temperature of the fluid at infinity

$C^{\prime} \quad$ Concentration of fluid near the plate

$C_{w}^{\prime} \quad$ Concentration of the fluid far away of the fluid from the plate

$C_{h}^{\prime} \quad$ Concentration of the fluid at infinity

$t^{\prime} \quad$ Time in $x^{\prime}, y^{\prime}$ coordinate system

Time in dimensionless co - ordinates

$u^{\prime} \quad$ Velocity component in $x^{\prime}-$ direction

$u$

Dimensionless velocity component in $x^{\prime}-$ direction

\section{Introduction:}

Natural convection flow induced by thermal and solutal buoyancy forces acting over bodies with different geometries in a fluid saturated porous medium is prevalent in many natural phenomena and has varied and wide range of industrial applications. For example, in atmospheric flows, the presence of pure air or water is impossible because some foreign mass may be present either naturally or mixed with air or water due to industrial emissions. Natural processes such as attenuation of toxic waste in water bodies, vaporization of mist and fog, photosynthesis, drying of porous solids, transpiration, sea - wind formation (where upward convection is modified by Coriolis forces), and formation of ocean currents [1] occur due to thermal and solutal buoyancy forces developed as a result of difference in temperature or concentration or a combination of these two. Such configuration is also encountered in several practical systems for industry based applications viz. heat exchanger devices, cooling of molten metals, insulation systems, petroleum reservoirs, filtration, chemical catalytic reactors and processes, nuclear waste repositories, desert coolers, wet bulb thermometers, frost formation in vertical channels, etc. Considering the importance of such fluid flow problems, extensive and in - depth research works have been carried out by several researchers [2-10] in the past. Comprehensive reviews of natural convection boundary layer flow over various geometrical bodies with heat and mass transfer in porous and nonporous media are well documented by Eckert and Drake [11], Gebhartet al. [12], Nield and Bejan [13], Pop and Ingham [14] and Incroperaet al. [15]. 
Coupled heat and mass transfer (or double - diffusion) driven by buoyancy, due to temperature and concentration variations in a saturated porous medium, has several important applications in geothermal and geophysical engineering such as the migration of moisture through the air contained in fibrous insulation, the extraction of geothermal energy, underground disposal of nuclear wastes, and the spreading of chemical contaminants through water - saturated soil. Bejan and Khair [16] investigated the vertical free convection boundary layer flow in porous media owing to combined heat and mass transfer. The suction and blowing effects on free convection coupled heat and mass transfer over a vertical plate in a saturated porous medium was studied by Raptiset al. [17] and Lai and Kulacki [18] respectively. Hydromagnetic flows and heat transfer have become more important in recent years because of its varied applications in agriculture, engineering and petroleum industries. Raptis [19] studied mathematically the case of time varying two - dimensional natural convective flow of an incompressible, electrically conducting fluid along an infinite vertical porous plate embedded in a porous medium. Soundalgekar [20] obtained approximate solutions for two - dimensional flow of an incompressible viscous flow past an infinite porous plate with constant suction velocity, the difference between the temperature of the plate and the free stream is moderately large causing free convection currents. Takhar and Ram [21] studied the MHD free convection heat transfer of water at $4^{\circ} \mathrm{C}$ through a porous medium. Soundalgekaret al. [22] analyzed the problem of free convection effects on Stokes problem for a vertical plate under the action of transversely applied magnetic field with mass transfer. Elbashbeshy [23] studied heat and mass transfer along a vertical plate under the combined buoyancy effects of thermal and species diffusion, in the presence of magnetic field.

In all these investigations, the radiation effects are neglected. For some industrial applications such as glass production and furnace design and in space technology applications, such as cosmical flight aerodynamics rocket, propulsion systems, plasma physics and spacecraft re - entry aerothermodynamics which operate at higher temperatures, radiation effects can be significant. Alagoaet al. [24] studied radiative and free convection effects on MHD flow through porous medium between infinite parallel plates with time - dependent suction. Bestman and Adjepong [25]analyzed unsteady hydromagnetic free convection flow with radiative heattransfer in a rotating fluid.Promise Mebine and Emmanue MunakuroghaAdigio [26]investigates the effects of thermal radiation on transientMHD free convection flow over a vertical surface embedded in a porousmedium with periodic temperature. Analytical solutions are obtainedfor the governing coupled dimensionless partial differential equations ofvelocity and temperature.An unsteady, two - dimensional, hydromagnetic, laminar free convective boundary - layer flow of an incompressible, Newtonian, electrically conducting and radiating fluid past an infinite heated verticalporous plate with heat and mass transfer is analyzed by Ramachandra Prasad and Bhaskar Reddy [27], by takinginto account the effect of viscous dissipation. The dimensionlessgoverning equations for this investigation are solved analyticallyusing two - term harmonic and non - harmonic functions. The influence of viscous dissipation and radiation on an unsteady MHD freeconvection flow past an infinite heatedvertical plate in a porous medium with time - dependent suction was studied by Israel - Cookeyet al.[28].

In fluid dynamics, Couette flow refers to the laminar flow of a viscous fluid in the space between two parallel plates, one of which moving relative to the other. The flow is driven by virtue of viscous drag force acting on the fluid and the applied pressure gradient parallel to the plates. This type of flow is named in honor of Maurice Marie Alfred Couette, a professor of physics at the French university of Angers in the late 19th century. Couette flow is frequently used in undergraduate physics and engineering courses to illustrate shear - driven fluid motion. Some important application areas of Couette motion are magnetohydrodynamics power generators and pumps, polymer technology, petroleum industry and purification of crude oil and fluid droplets sprays. Singh [29] studied unsteady free convection flow of an incompressible viscous fluid between two vertical parallel plates, in which one is fixed and other is impulsively started in its own plane. Kearsley [30] studied problem of steady state Couette flow with viscous heating. MHD free convection Couette flow between two vertical parallel plates has been studied by Jha [31]. Mebine[32] studied the effect of thermal radiation on MHD Couette flow with heat transfer between two parallel plates. The natural convection in unsteady Couette flow of a viscous incompressible fluid confined between two vertical parallel plates in the presence of thermal radiation has been studied by Narahari [33]. Seth et al.[34] have studied unsteady MHD Couette flow of a viscous incompressible electrically conducting fluid, in the presence of a transverse magnetic field, between two parallel porous plates. Deka and Bhattacharya [35] obtained an exact solution of unsteady free convective Couette flow of a viscous incompressible heat generating or absorbing fluid confined between two vertical plates in a porous medium. Rajput and Sahu [36] found the exact solution of natural convection in unsteady hydromagneticCouette flow of a viscous incompressible electrically conducting fluid between two vertical parallel plates in the presence of thermal radiation is obtained here. The magnetic lines of force are assumed to be fixed relative to the moving plate. The fluid is considered to be a gray, absorbing-emitting but non - scattering medium, and the Rosseland approximation is used to describe the radiative heat flux in the energy equation. The non - dimensional governing equations involved in the present analysis are solved using the Laplace transform technique.

The objective of the present chapter is to study the radiation, heat and mass transfer effects on an unsteady two - dimensional natural convective Couetteflow of a viscous, incompressible, electrically conducting fluid between two parallel plates with suction, embedded in a porous medium, under the influence of a uniform transverse magnetic field. The problemis described by a system of coupled nonlinear partial differential equations, whose exact solutions are difficult to obtain,whenever possible. Thus, the finite difference method is adopted for the solution, which is more economicalfrom a computational point of view. The behavior of the velocity, temperature, concentration, skin - friction coefficient, Nusselt number and Sherwood number has been discussed in detail for variations in the physical parameters. In section 2 , the mathematical formulation of the problem and dimension less forms of the governing equations are established. Solution method tothese equations for the flow variables are briefly examined in section 3 . Theresults of the previous sections are discussed in section 4 . In section 5 , generalconcluding remarks of the results of the previous sections are given. 


\section{Mathematical formulation:}

Consider the unsteady two - dimensional natural convective Couette flow of a viscous, incompressible, electrically conducting fluid past vertical a heated porous plate with suction, under the influence of a uniform transverse magnetic field, thermal radiation, heat and mass transfer as shown by figure -1 . We made the following assumptions.

1. The $x^{\prime}$-axis is taken along the plate in the vertical upward direction and the $y^{\prime}$-axis is taken normal to the plate.

2. Let the plates are separated by a distance $h$. Initially, at time $t^{\prime} \leq 0$, the fluid and the plates of the channel are assumed to be at rest and at same temperature $T_{h}^{\prime}$ and concentration $C_{h}^{\prime}$.

3. When time $t^{\prime}>0$, the plate (at $y^{\prime}=0$ ) starts moving with time dependent velocity $U_{o} t^{\prime n}$ ( $U_{o}$ being a constant and $n$ being a non - negative integer) in its own plane and at the same time the plate temperature is raised to $T_{w}^{\prime}$ while the plate (at $y^{\prime}=h$ ) is kept fixed.

4. A transverse magnetic field of uniform strength $H_{o}$ is assumed to be applied normal to the plate.

5. The induced magnetic field, Hall Effect and viscous dissipation are assumed to be negligible as the magnetic Reynolds number of the flow is taken to be very small.

6. It is assumed that there is no applied voltage which implies the absence of an electric field.

7. The homogeneous chemical reaction is of first order with rate constant $\bar{K}$ between the diffusing species and the fluid is neglected.

8. The fluid has constant kinematic viscosity and constant thermal conductivity and the Boussinesq's approximation have been adopted for the flow.

9. The concentration of the diffusing species in the binary mixture is assumed to be very small in comparison with the other chemical species, which are present and hence Soret and Dufour effects are negligible. 


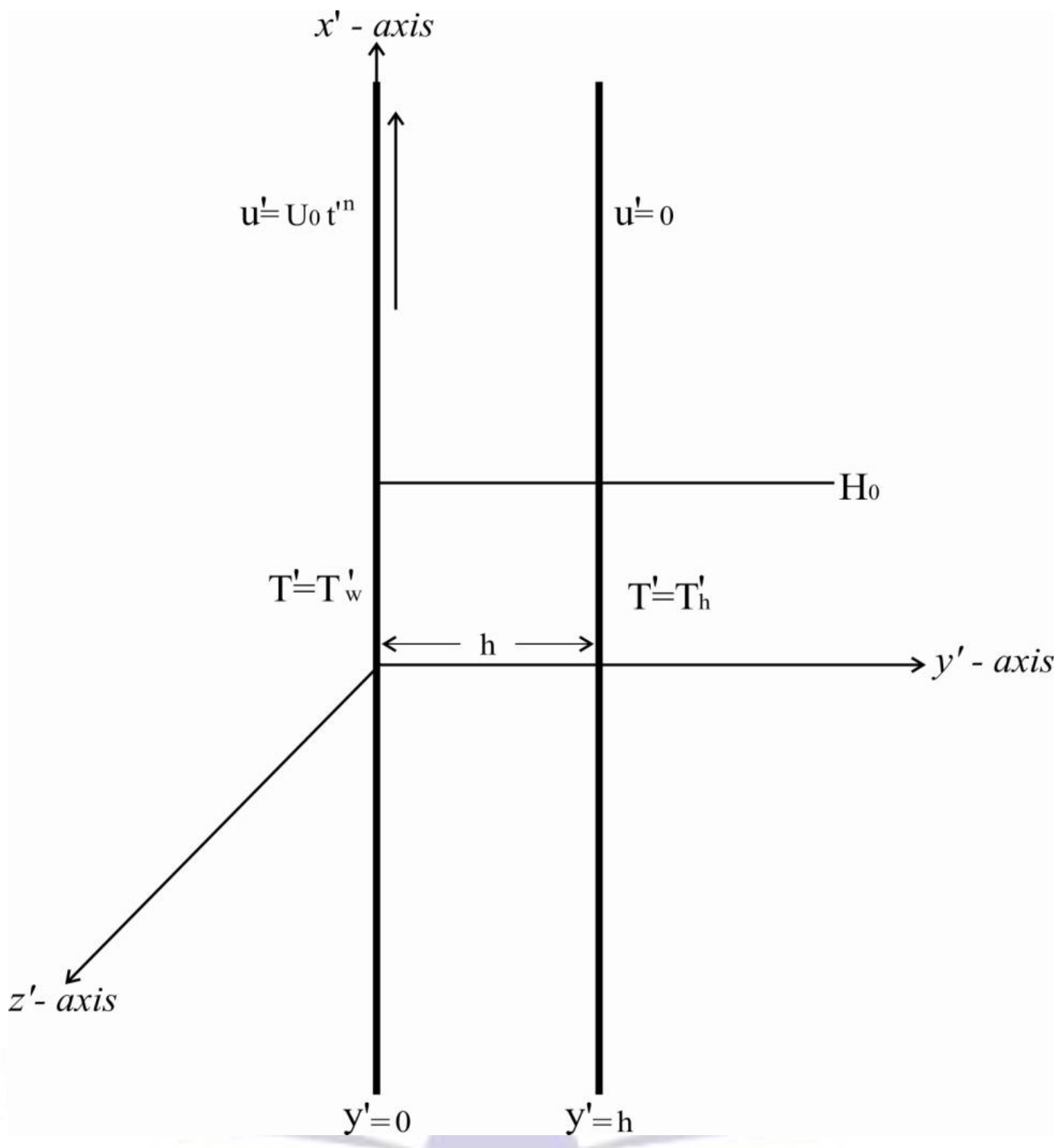

Figure 1. Physical Model in the problem

Then by usual Boussinesq's approximation, the unsteady flow is governed by the following equations.

Momentum Equation:

$\frac{\partial u^{\prime}}{\partial t^{\prime}}=v \frac{\partial^{2} u^{\prime}}{\partial y^{\prime 2}}+g \beta\left(T^{\prime}-T_{\infty}^{\prime}\right)+g \beta^{*}\left(C^{\prime}-C_{\infty}^{\prime}\right)-\frac{\sigma u_{e}^{2} H_{o}^{2}}{\rho}\left(u^{\prime}-U_{o} t^{\prime n}\right)(1)$

Energy Equation:

$\rho C_{p} \frac{\partial T^{\prime}}{\partial t^{\prime}}=\kappa \frac{\partial^{2} T^{\prime}}{\partial y^{\prime 2}}-\frac{\partial q_{r}}{\partial y}(2)$

Species Diffusion Equation: 
$\frac{\partial C^{\prime}}{\partial t^{\prime}}=D \frac{\partial^{2} C^{\prime}}{\partial y^{\prime 2}}(3)$

The initial and boundary conditions are

$t^{\prime} \leq 0: u^{\prime}=0, T^{\prime}=T_{h}^{\prime}, C^{\prime}=C_{h}^{\prime}$ for $0 \leq y^{\prime} \leq h$
$t^{\prime}>0:\left\{\begin{array}{l}u^{\prime}=U_{o} t^{\prime n}, T^{\prime}=T_{w}^{\prime}, C^{\prime}=C_{w}^{\prime} \text { at } y^{\prime}=0 \\ u^{\prime}=0, T^{\prime}=T_{h}^{\prime}, C^{\prime}=C_{h}^{\prime} \text { at } y^{\prime}=h\end{array}\right\}$

The radiative heat flux term is simplified by making use of the Rosseland approximation [37] as $q_{r}=-\frac{4 \bar{\sigma}}{3 k^{*}} \frac{\partial T^{\prime 4}}{\partial y^{\prime}}$

Here $\bar{\sigma}$ is Stefan - Boltzmann constant and $k^{*}$ is the mean absorption coefficient. It is assumed that the temperature differences within the flow are sufficiently small so that $T^{\prime 4}$ can be expressed as a linear function of $T^{\prime}$ after using Taylor's series to expand $T^{\prime 4}$ about the free stream temperature $T_{h}^{\prime}$ and neglecting higher - order terms. This results in the following approximation:

$T^{\prime 4} \cong 4 T_{h}^{\prime 3} T^{\prime}-3 T_{h}^{\prime 4}$

Using equations (5) and (6) in the last term of equation (2), we obtain:

$\frac{\partial q_{r}}{\partial y^{\prime}}=-\frac{16 \bar{\sigma} T_{h}^{\prime 3}}{3 k^{*}} \frac{\partial^{2} T^{\prime}}{\partial y^{\prime 2}}$

Introducing (7) in the equation (2), the energy equation becomes:

$\rho C_{p} \frac{\partial T^{\prime}}{\partial t^{\prime}}=\kappa \frac{\partial^{2} T^{\prime}}{\partial y^{\prime 2}}+\frac{16 \bar{\sigma} T_{h}^{3}}{3 k^{*}} \frac{\partial^{2} T^{\prime}}{\partial y^{\prime 2}}$

To find the solutions of equations (1), (3) and (8) subject to the initial and boundary conditions (3),two cases are considered:

1. Impulsive movement of the plate at $y^{\prime}=0$ (i.e. $n=0$ ) and

2. Uniformly accelerated movement of the plate at $y^{\prime}=0$ (i.e. $n=1$ ).

Case - 1: Impulsive movement of the plate $y^{\prime}=0$ :

Taking $n=0$ in equation (1) and introducing the following non - dimensional quantities in equations (1), (3) and (8):

$$
\begin{aligned}
& u=\frac{u^{\prime}}{h}, y=\frac{y^{\prime}}{h}, t=\frac{t^{\prime} v}{h^{2}}, \theta=\frac{T^{\prime}-T_{h}^{\prime}}{T_{w}^{\prime}-T_{h}^{\prime}}, \phi=\frac{C^{\prime}-C_{h}^{\prime}}{C_{w}^{\prime}-C_{h}^{\prime}}, G r=\frac{g \beta h^{2}\left(T_{w}^{\prime}-T_{h}^{\prime}\right)}{v U_{o}}, \\
& \left.G c=\frac{g \beta^{*} h^{2}\left(C_{w}^{\prime}-C_{h}^{\prime}\right)}{v U_{o}}, M^{2}=\frac{\sigma \mu_{e}^{2} H_{o}^{2} h^{2}}{\rho v}, R=\frac{\kappa k^{*}}{4 \sigma T_{h}^{\prime 3}}, \operatorname{Pr}=\frac{\mu C_{p}}{\kappa}, S c=\frac{v}{D}, F=\frac{h^{2}}{v}\right\}
\end{aligned}
$$

The equations (1), (3) and (8) reduce to the following non - dimensional form of equations:

$$
\begin{aligned}
& \frac{\partial u}{\partial t}=\frac{\partial^{2} u}{\partial y^{2}}+G r \theta+G c \phi-M^{2}(u-1) \\
& \frac{\partial \theta}{\partial t}=\frac{1}{\operatorname{Pr}}\left(\frac{3 R+4}{3 R}\right) \frac{\partial^{2} \theta}{\partial y^{2}}
\end{aligned}
$$

(11) $\frac{\partial \phi}{\partial t}=\frac{1}{S c} \frac{\partial^{2} \phi}{\partial y^{2}}$

The initial and boundary conditions (4)reduce to: 
$t \leq 0: u=0, \theta=0, \phi=0$ for $0 \leq y \leq 1$
$t>0:\left\{\begin{array}{l}u=1, \theta=1, \phi=1 \text { at } y=0 \\ u=0, \theta=0, \phi=0 \text { at } y=1\end{array}\right\}$

Case - 2: Uniformly accelerated movement of the plate (at $y^{\prime}=0$ )

Taking $n=1$ in equation (1) and using equation (9), the equation (1) reduces to the following non - dimensional form of equation:

$$
\frac{\partial u}{\partial t}=\frac{\partial^{2} u}{\partial y^{2}}+G r \theta+G c \phi-M^{2}(u-F t)
$$

The initial and boundary conditions (4)reduce to:

$$
\begin{aligned}
& t \leq 0: u=0 \text { for } 0 \leq y \leq 1 \\
& t>0:\left\{\begin{array}{l}
u=F t \text { at } y=0 \\
u=0 \text { at } y=1
\end{array}\right\}
\end{aligned}
$$

For practical engineering applications and the design of chemical engineering systems, quantities of interest include the following Skin - friction, Nusselt number and Sherwood number are useful to compute.

Skin - friction: The skin - friction or the shear stress at the moving plate of the channel in non - dimensional form is given by:

$$
\tau_{1}=-\left[\frac{\partial u}{\partial y}\right]_{y=0}
$$

Nusselt Number: The rate of heat transfer at the moving hot plate of the channel in non - dimensional form is given by:

$$
N u_{0}=-\left[\frac{\partial \theta}{\partial y}\right]_{y=0} \text { (17) }
$$

Further the rate of heat transfer on the stationary plate is given by:

$$
N u_{1}=-\left[\frac{\partial \theta}{\partial y}\right]_{y=1}
$$

Sherwood number: The Sherwood number at the moving plate of the channel in non - dimensional form is given by:

$S h=-\left[\frac{\partial \phi}{\partial y}\right]_{y=0}$

The mathematical formulation of the problem is now completed. Equations (10)-(12)\& (14) present a coupled nonlinear system of partial differential equations and are to be solved by using initial and boundary conditions (13)\& (15). However, exact solutions are difficult, whenever possible. Hence, these equations are solved by the Crank Nicholson method.

\section{Numerical Solution by Crank Nicholson Method:}

Equations (10) - (12) \& (14) represent coupled system of non - linear partial differential equations which are solved numerically under the initial and boundary conditions (13) \&(15) using the finite difference approximations. A linearization technique is first applied to replace the non - linear terms at a linear stage, with the corrections incorporated in subsequent iterative steps until convergence is reached. Then the Crank - Nicolson implicit method is used at two successive time levels [38]. An iterative scheme is used to solve the linearized system of difference equations. The solution at a certain time step is chosen as an initial guess for next time step and the iterations are continued till convergence, within a prescribed accuracy. Finally, the resulting block tri diagonal system is solved using the generalized Thomas - algorithm [38]. Finite difference equations relating the variables are obtained by writing the equations at the midpoint of the computational cell and then replacing the different terms by their second order central difference approximations in the $y$-direction. The diffusion terms are replaced by the average of the central differences at two successive time - levels. The computational domain is divided into meshes of dimension $\Delta t$ and $\Delta y$ in time and space respectively as shown in figure 2. We define the variables $B=u_{y}, L=\theta_{y}$ and $Z=\phi_{y}$ to reduce the second order 
differential equations (10) - (12) to first order differential equations. The finite difference representations for the resulting first order differential equations (10) - (12)\& (14)take the following forms:

$$
\begin{aligned}
& \left(\frac{u_{i+1, j+1}-u_{i, j+1}+u_{i+1, j}-u_{i, j}}{2(\Delta t)}\right)=\left(\frac{\left(B_{i+1, j+1}+B_{i, j+1}\right)-\left(B_{i+1, j}+B_{i, j}\right)}{2(\Delta y)}\right)+G r\left(\frac{\theta_{i+1, j+1}+\theta_{i, j+1}+\theta_{i+1, j}+\theta_{i, j}}{4}\right) \\
& +G c\left(\frac{\phi_{i+1, j+1}+\phi_{i, j+1}+\phi_{i+1, j}+\varphi_{i, j}}{4}\right)-M^{2}\left(\frac{u_{i+1, j+1}+u_{i, j+1}+u_{i+1, j}+u_{i, j}}{4}\right)+M^{2}
\end{aligned}
$$

$\left(\frac{\theta_{i+1, j+1}-\theta_{i, j+1}+\theta_{i+1, j}-\theta_{i, j}}{2(\Delta t)}\right)=\frac{1}{\operatorname{Pr}}\left(\frac{3 R+4}{3 R}\right)\left(\frac{\left(L_{i+1, j+1}+L_{i, j+1}\right)-\left(L_{i+1, j}+L_{i, j}\right)}{4}\right)$

$\left(\frac{\phi_{i+1, j+1}-\phi_{i, j+1}+\phi_{i+1, j}-\phi_{i, j}}{2(\Delta t)}\right)=\frac{1}{S c}\left(\frac{\left(Z_{i+1, j+1}+Z_{i, j+1}\right)-\left(Z_{i+1, j}+Z_{i, j}\right)}{4}\right)$

$\left(\frac{u_{i+1, j+1}-u_{i, j+1}+u_{i+1, j}-u_{i, j}}{2(\Delta t)}\right)=\left(\frac{\left(B_{i+1, j+1}+B_{i, j+1}\right)-\left(B_{i+1, j}+B_{i, j}\right)}{2(\Delta y)}\right)+G r\left(\frac{\theta_{i+1, j+1}+\theta_{i, j+1}+\theta_{i+1, j}+\theta_{i, j}}{4}\right)$

$+G c\left(\frac{\phi_{i+1, j+1}+\phi_{i, j+1}+\phi_{i+1, j}+\varphi_{i, j}}{4}\right)-M^{2}\left(\frac{u_{i+1, j+1}+u_{i, j+1}+u_{i+1, j}+u_{i, j}}{4}\right)+M^{2} F t$

$y(i, j+1)(i+1, j+1)$

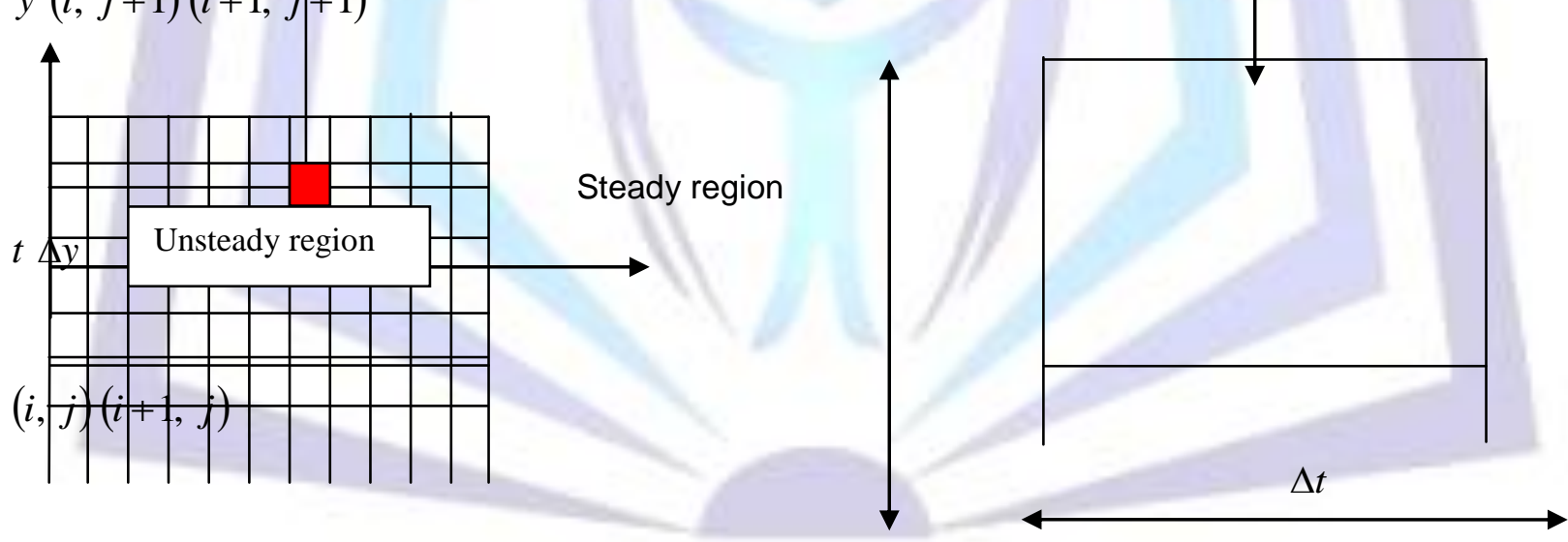

Figure 2. Mesh Layout

Computations have been made for $G r=2.0, G c=2.0, \operatorname{Pr}=0.71, S c=0.22, R=2.0, M=2.0$,

$F=$ 0.3 and $t=0.1$. Grid - independence studies show that the computational domain $0<t<\infty$ and $-1<y<1$ can be divided into intervals with step sizes $\Delta t=0.0001$ and $\Delta y=0.005$ for time and space respectively. The truncation error of the central difference schemes of the governing equations is $O\left(\Delta t^{2}, \Delta y^{2}\right)$. Stability and rate of convergence are functions of the flow and heat parameters. Smaller step sizes do not show any significant change in the results. Convergence of the scheme is assumed when all of the unknowns $u, \theta$ and $\phi$ for the last two approximations differ from unity by less than $10^{-6}$ for all values of $y$ in $-1<y<1$ at every time step. Less than 7 approximations are required to satisfy this convergence criteria forall ranges of the parameters studied here.

\section{Results and Discussions:}


To study the effects of different parameters like thermal Grashof number $G r$, solutalGrashof number $G c$, magnetic parameter $M$, thermal radiation parameter $R$, Prandtl number $\operatorname{Pr}$, Schmidt number $S c$, accelerating parameter $F$ and time $t$ on the flow field the numerical results of the fluid velocity, temperature, concentration, skin friction, Nusselt number and Sherwood number are computed numerically by applying Finite difference method in both the cases viz.

(I) Impulsive movement of the plate (at $y^{\prime}=0$ ) and

(II) Uniformly accelerated movement of the plate (at $y^{\prime}=0$ ).

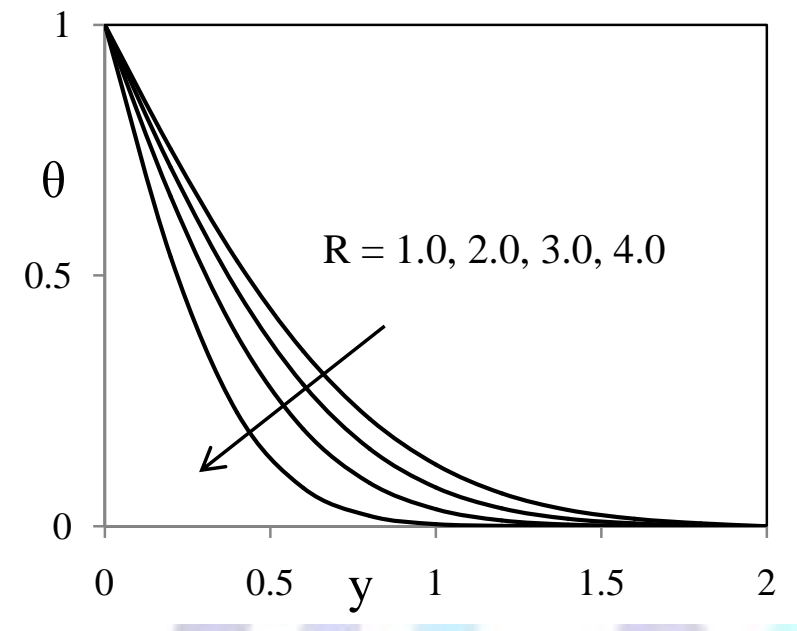

Figure 3: Temperature Profiles for different values of $R$

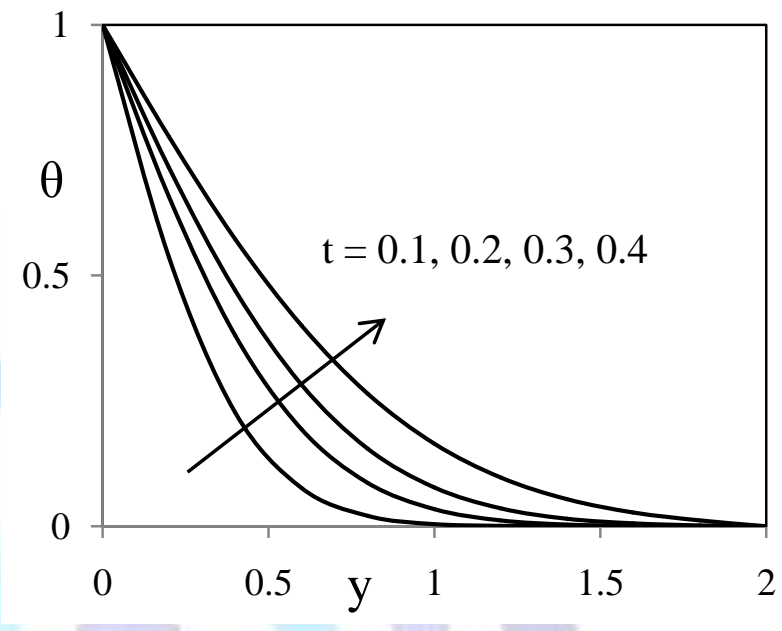

Figure 4: Temperature Profiles for different values of $t$

The values of the main parameters considered are: magnetic parameter $M=2.0,4.0,6.0,8.0$; radiation parameter $R=$ 1.0, 2.0, 5.0, 10.0; Accelerating parameter $F=0.3,0.5,0.7,0.9$; time $t=0.1,0.2,0.3,0.4$; Grashof number $G r=2.0$, 5.0, 7.0, 10.0; solutalGrashof number $G c=2.0,5.0,7.0,10.0$; PrandtInumber $\operatorname{Pr}=0.025$, (Mercury), 0.71 (for air), 3.0 (for the saturated liquid Freon at $273.3 \mathrm{~K}$ ) and 7.0 (for water); Schmidt number $S c=0.22$ (Hydrogen), 0.30 (Helium), 0.60 (Water vapor), 0.66 (Oxygen) and 0.78 (Ammonia).

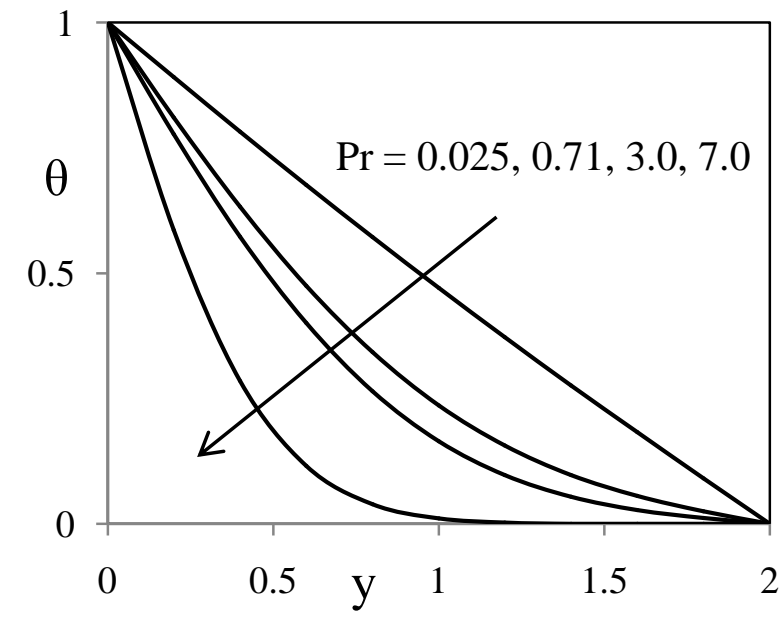

Figure 5: Temperature Profiles for different values of $\mathrm{Pr}$

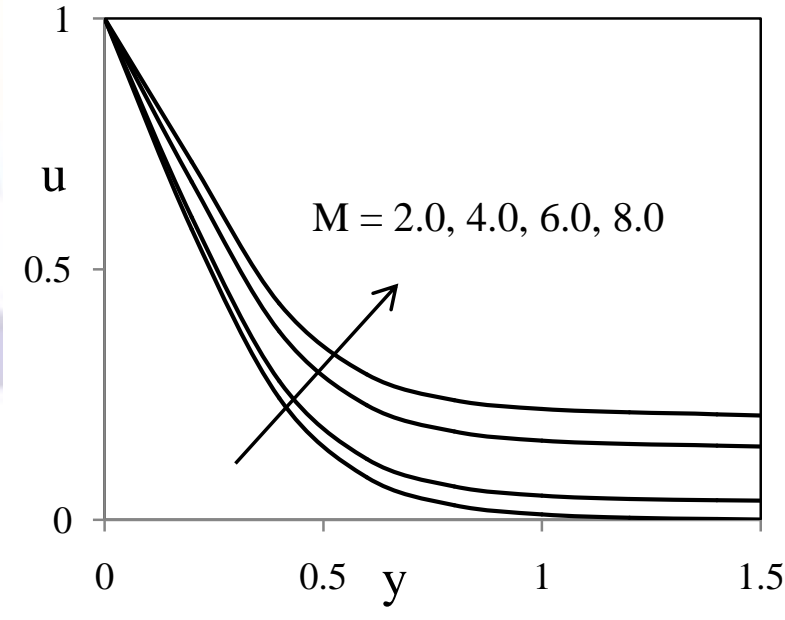

Figure 6: Velocity Profiles for different values of $M$ in case of impulsive movement of the plate

Figures (3) - (5) show the effects of radiation parameter, time and Prandtl number on the temperature of fluid, respectively. The effects of the Prandtl number and radiation parameter play an important role in temperature field. It is clear that temperature decreases as the Prandtl number $\operatorname{Pr}$ increases. This shows that the heat transfer is more in air than in water and Gasoline. Further, it is seen that the temperature decreases as the radiation parameter $R$ increases. This result qualitatively agrees with expectations, since the effect of radiation is to decrease the rate of energy transport to the fluid, thereby decreasing the temperature of the fluid. The fluid temperature increases with increasing time $t$. Hence it 
is clear from these figures that the temperature increases, with maximum at the plate $(y=0)$ and minimum at the plate ( $y=1$ ).Figures (6) and (7) display the effects of $M$ (magnetic parameter) on the velocity field. It is observed that velocity of fluid increases with increasing magnetic parameter (In case of impulsive movement of the plate) but decreases with increasing magnetic parameter near the moving plate (In case of uniformly accelerated movement of the plate). Figures (8) and (9) represent the velocity profiles for different values of thermal Grashof number $G r$. It is observed that the velocity of fluid increases with increasing thermal Grashof number in both the cases. Further, as we move far away from the moving plate the effect of the thermal Grashof number is not found to be significant. The different values of solutal Grashof number $G c$, the velocity profiles in the boundary layer are shown in figures (10) and (11) in both the cases I and II. The velocity distribution attains a distinctive maximum value in the vicinity of the plate and then decreases properly to approach a free stream value. As expected, the fluid velocity increases and the peak value becomes more distinctive due to increase in the buoyancy force represented by $G c$. Figures (12) and (13) illustrate the influences of $t$ (time) on the velocity profiles. From these figures, the velocity is found to increase with increase in $t$ (time) in both the cases.

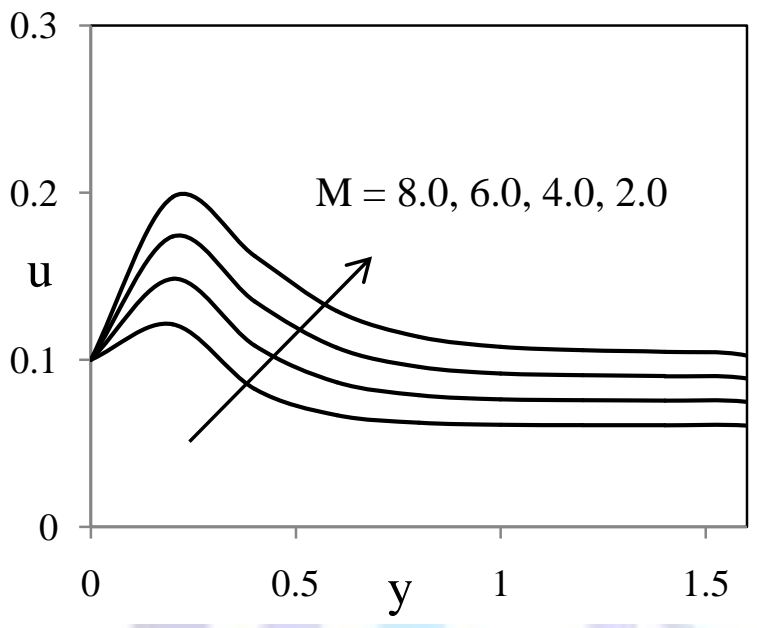

Figure 7: Velocity Profiles for different values of $M$ in case of uniformly accelerated movement of the plate

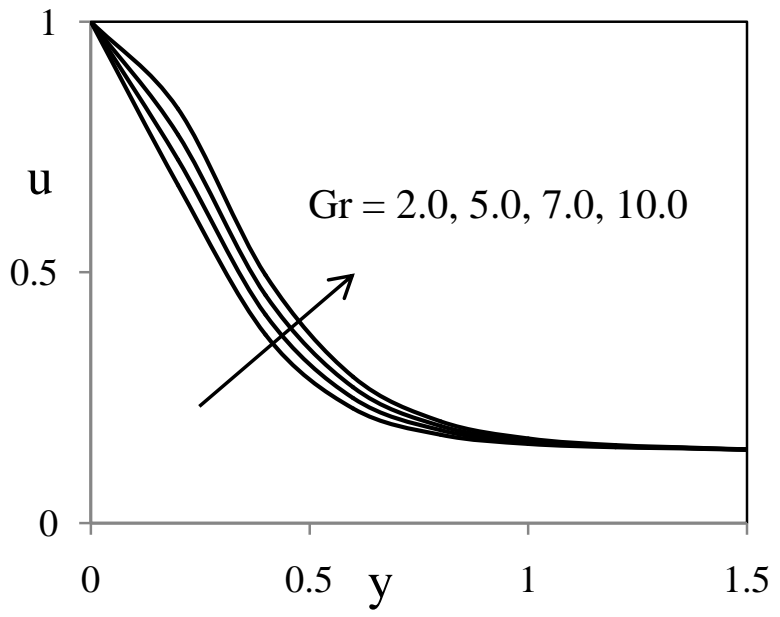

Figure 8: Velocity Profiles for different values of $G r$ in case of impulsive movement of the plate

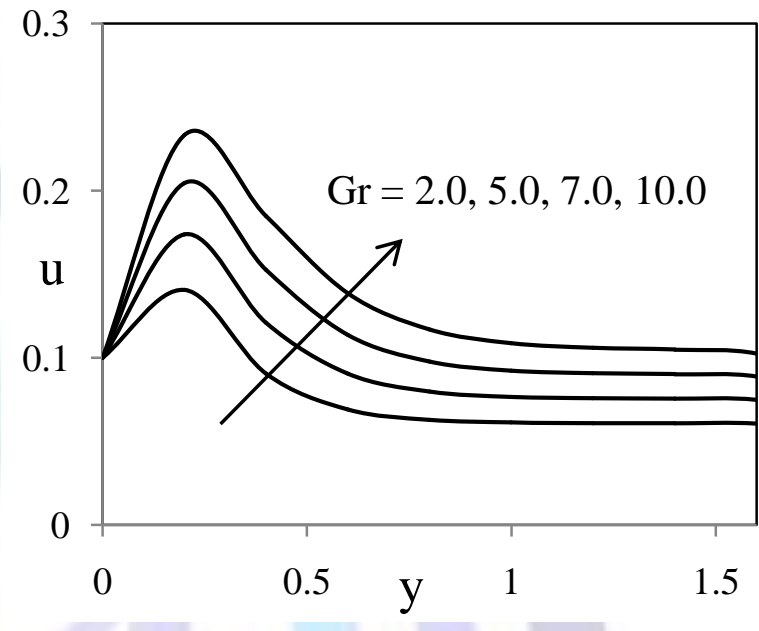

Figure 9: Velocity Profiles for different values of $G r$ in case of uniformly accelerated movement of the plate

$$
1
$$

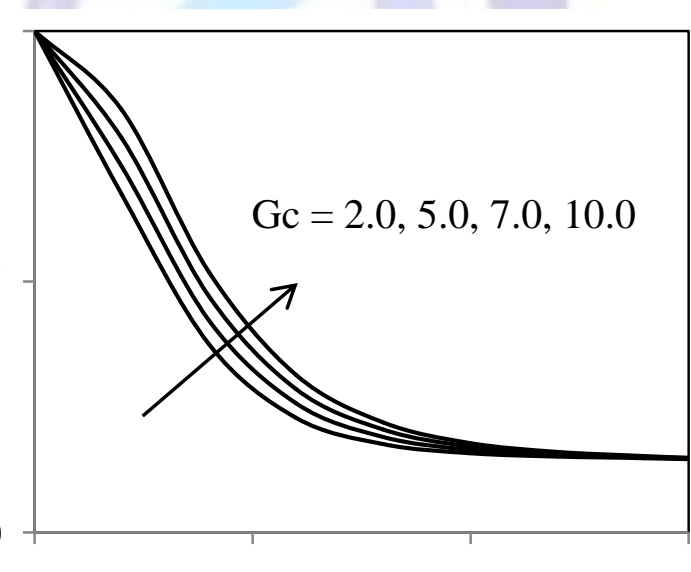

Figure 10: Velocity Profiles for different values of $G c$ in case of impulsive movement of the plate

Figures (14) and (15) represent the velocity profiles for different values of $R$ (radiation parameter). From these figures, it is observed that the fluid velocity decreases with increasing radiation parameter in both the cases. This is in agreement with the fact that the thermal boundary layer thickness decreases with increasing radiation parameter. 


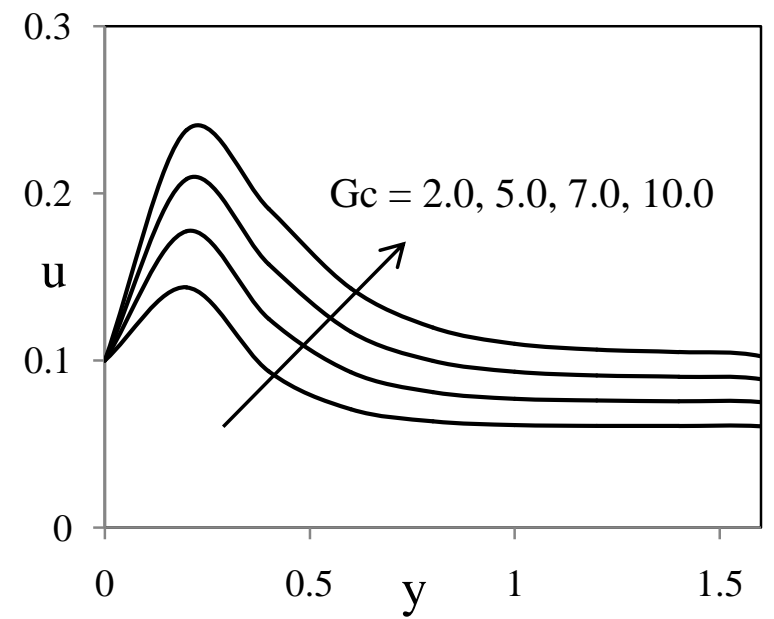

Figure 11: Velocity Profiles for different values of $G c$ in case of uniformly accelerated movement of the plate

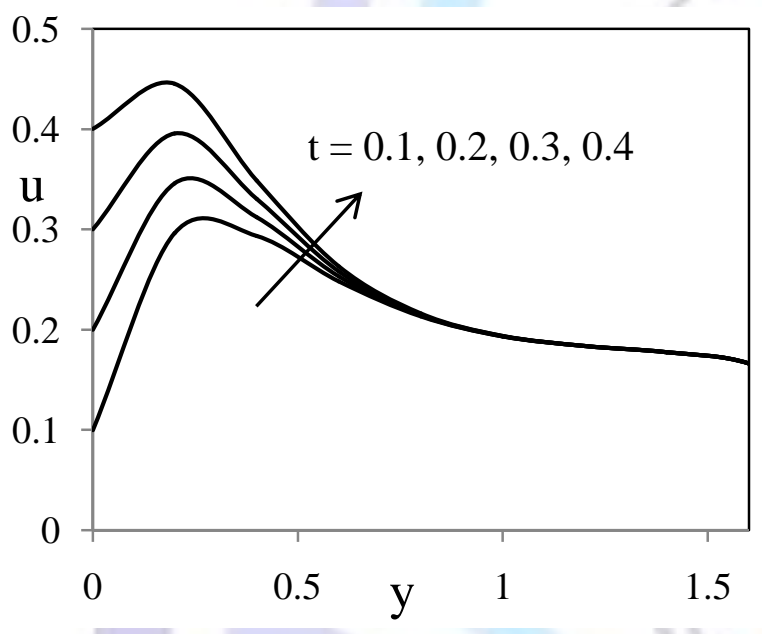

Figure 13: Velocity Profiles for different values of $t$ in case of uniformly accelerated movement of the plate

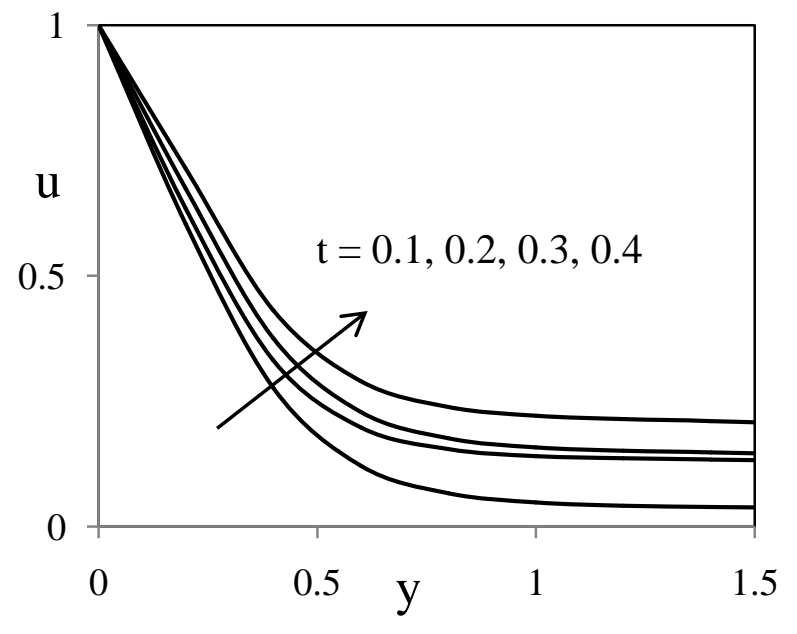

Figure 12: Velocity Profiles for different values of $t$ in case of impulsive movement of the plate

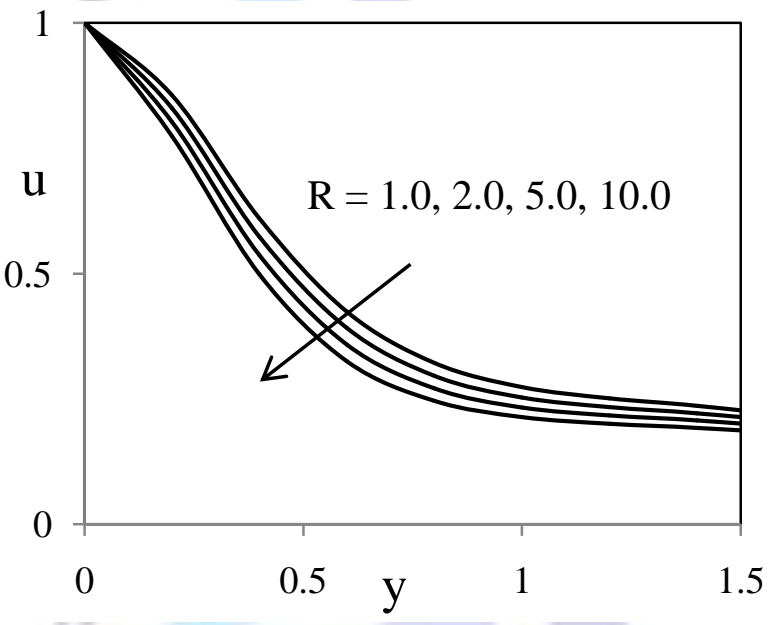

Figure 14: Velocity Profiles for different values of $R$ in case of impulsive movement of the plate

Figures (16) and (17) display the effects of Pr (Prandtl number) on the velocity profiles. From these figures, it is clear that the velocity of fluid decreases as the value of Prandtl number increases in both the cases. Physically this is possible because fluids with high Prandtl number have greater viscosity, which makes the fluid thick and hence move slowly. Figure (18) represents velocity profiles for various values of the accelerating parameter $(F=0.3,0.5,0.7,0.9)$. This shows that the velocity increases with increasing accelerating parameter $F$. Further, as we move far away from the moving plate the effect of accelerating parameter is almost found to be nil. Also, near the moving plate the fluid velocity is maximum but near the fixed plate the fluid velocity is minimum. 


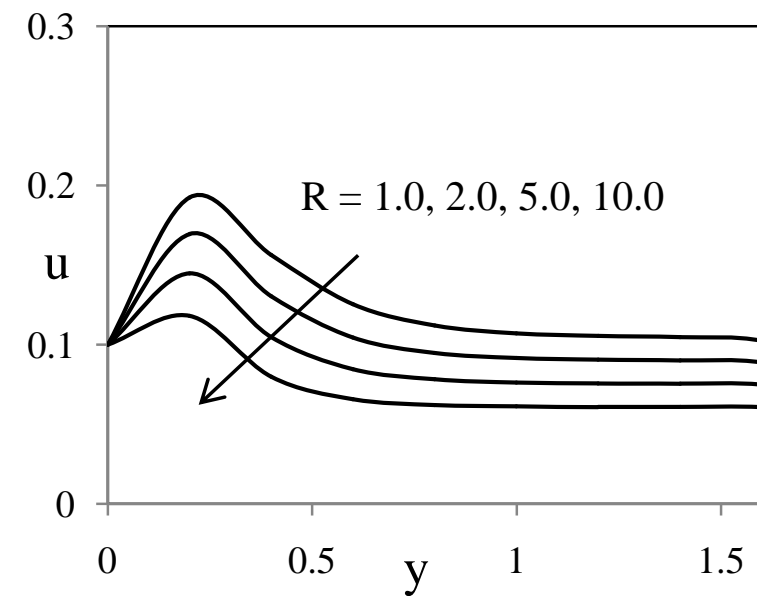

Figure 15: Velocity Profiles for different values of $R$ in case of uniformly accelerated movement of the plate

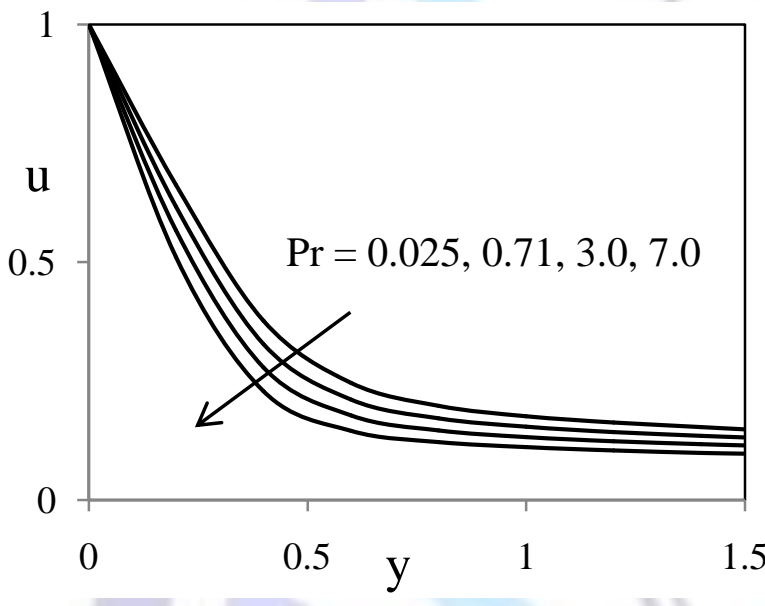

Figure 16: Velocity Profiles for different values of $\mathrm{Pr}$ in case of impulsive movement of the plate

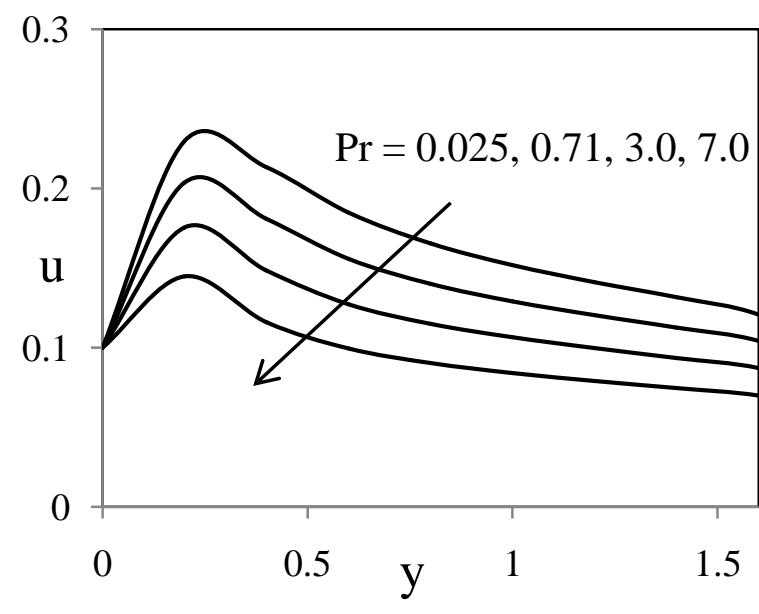

Figure 17: Velocity Profiles for different values of $\mathrm{Pr}$ in case of uniformly accelerated movement of the plate

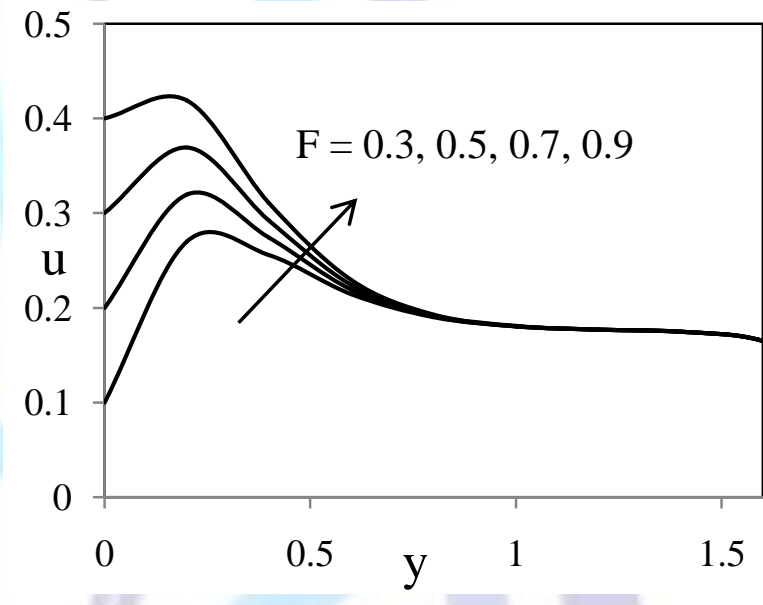

Figure 18: Velocity Profiles for different values of $F$ in case of uniformly accelerated movement of the plate

The effect of Schmidt number $S c$ on the velocity and concentration are shown in figures (19) - (21) in both the cases I and II. As the Schmidt number increases, the velocity and concentration decreases in both the cases I and II. This causes the concentration buoyancy effects to decrease yielding a reduction in the fluid velocity. Reductions in the velocity and concentration distributions are accompanied by simultaneous reductions in the velocity and concentration boundary layers. Table -1 shows the effects of thermal Grashof number, solutalGrashof number, Prandtl number, Schmidt number, Magnetic parameter, Thermal radiation parameter, Accelerating parameter and time $t$ in case I. From this table, it is observed that the skin - friction decreases with increasing magnetic parameter, Schmidt number, thermal Grashof number and solutalGrashof number and increases with increasing radiation parameter, Prandtl number, Accelerating parameter and time $t$. 


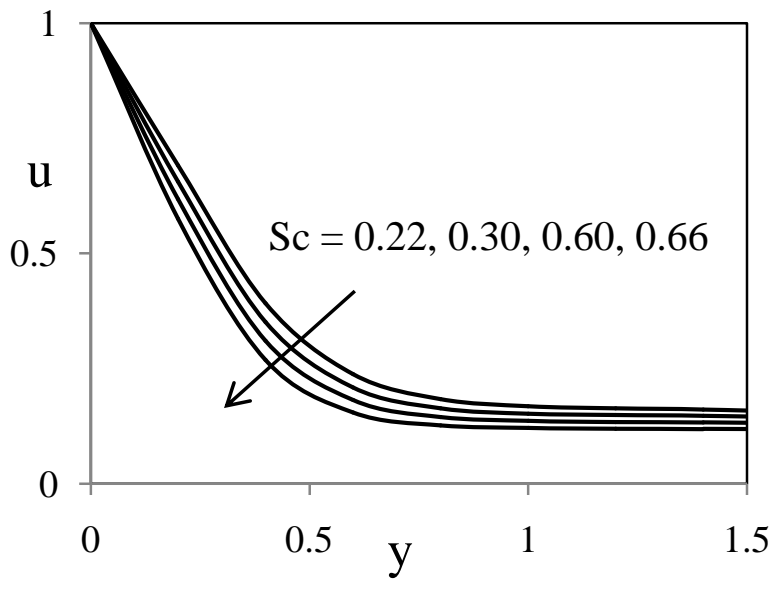

Figure 19: Velocity Profiles for different values of $S c$ in case of impulsive movement of the plate

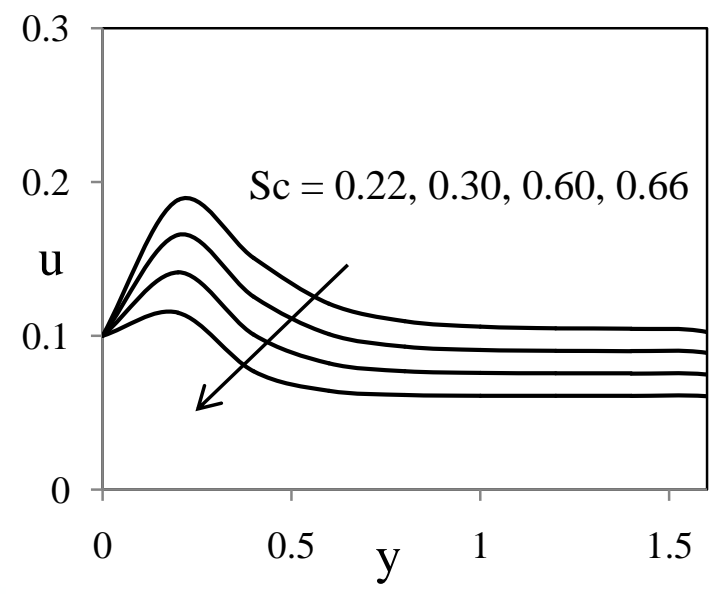

Figure 20: Velocity Profiles for different values of $S c$ in case of uniformly accelerated movement of the plate

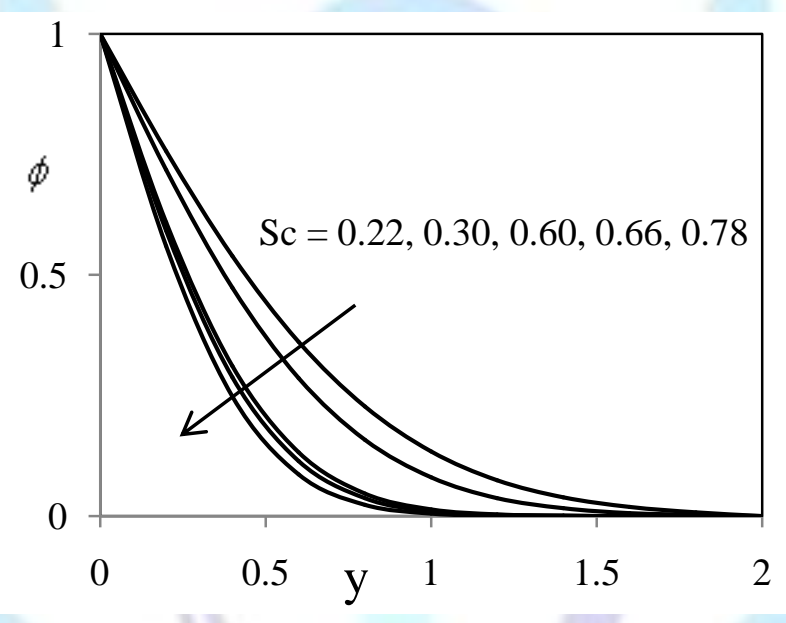

Figure 21: Concentration Profiles for different values of $S c$

Table -2 shows the effects of thermal Grashof number, solutalGrashof number, Prandtl number, Schmidt number, Magnetic parameter, Thermal radiation parameter, Accelerating parameter and time $t$ in case II. From this table, it is observed that the skin - friction decreases with increasing magnetic parameter, Schmidt number, thermal Grashof number and solutalGrashof number and increases with increasing radiation parameter, Prandtl number, Accelerating parameter and time $t$.

Table - 1: Skin - friction in case of impulsive movement of the plate

\begin{tabular}{|c|c|c|c|c|c|c|c|}
\hline$G r$ & $G c$ & $\operatorname{Pr}$ & $S c$ & $M$ & $R$ & $t$ & $\tau_{o}$ \\
\hline 5.0 & 5.0 & 0.71 & 0.22 & 2.0 & 1.0 & 0.1 & 4.025614 \\
\hline 10.0 & 5.0 & 0.71 & 0.22 & 2.0 & 1.0 & 0.1 & 5.136942 \\
\hline 5.0 & 10.0 & 0.71 & 0.22 & 2.0 & 1.0 & 0.1 & 5.559808 \\
\hline 5.0 & 5.0 & 10.0 & 0.22 & 2.0 & 1.0 & 0.1 & 3.841329 \\
\hline 5.0 & 5.0 & 0.71 & 0.30 & 2.0 & 1.0 & 0.1 & 3.752164 \\
\hline 5.0 & 5.0 & 0.71 & 0.22 & 4.0 & 1.0 & 0.1 & 4.159732 \\
\hline 5.0 & 5.0 & 0.71 & 0.22 & 2.0 & 2.0 & 0.1 & 3.619873 \\
\hline 5.0 & 5.0 & 0.71 & 0.22 & 2.0 & 1.0 & 0.2 & 4.268596 \\
\hline
\end{tabular}


Table - 2: Skin - friction in case of uniformly accelerated movement of the plate

\begin{tabular}{|c|c|c|c|c|c|c|c|c|}
\hline$G r$ & $G c$ & $\operatorname{Pr}$ & $S c$ & $M$ & $R$ & $t$ & $F$ & $\tau_{o}$ \\
\hline 5.0 & 5.0 & 0.71 & 0.22 & 2.0 & 1.0 & 0.1 & 0.5 & -0.541291 \\
\hline 10.0 & 5.0 & 0.71 & 0.22 & 2.0 & 1.0 & 0.1 & 0.5 & -0.487215 \\
\hline 5.0 & 10.0 & 0.71 & 0.22 & 2.0 & 1.0 & 0.1 & 0.5 & -0.401259 \\
\hline 5.0 & 5.0 & 10.0 & 0.22 & 2.0 & 1.0 & 0.1 & 0.5 & -0.614743 \\
\hline 5.0 & 5.0 & 0.71 & 0.30 & 2.0 & 1.0 & 0.1 & 0.5 & -0.629884 \\
\hline 5.0 & 5.0 & 0.71 & 0.22 & 4.0 & 1.0 & 0.1 & 0.5 & -0.448337 \\
\hline 5.0 & 5.0 & 0.71 & 0.22 & 2.0 & 2.0 & 0.1 & 0.5 & -0.602121 \\
\hline 5.0 & 5.0 & 0.71 & 0.22 & 2.0 & 1.0 & 0.2 & 0.5 & -0.443252 \\
\hline 5.0 & 5.0 & 0.71 & 0.22 & 2.0 & 1.0 & 0.1 & 0.9 & -0.422663 \\
\hline
\end{tabular}

Table - 3: Nusselt Number

\begin{tabular}{|c|c|c|c|c|}
\hline $\operatorname{Pr}$ & $R$ & $t$ & $N u_{o}$ & $N u_{1}$ \\
\hline 0.71 & 1.0 & 0.1 & 1.589244 & 0.096653 \\
\hline 10.0 & 1.0 & 0.1 & 1.501475 & 0.091546 \\
\hline 0.71 & 2.0 & 0.1 & 1.514696 & 0.092019 \\
\hline 0.71 & 1.0 & 0.2 & 1.805483 & 0.102688 \\
\hline
\end{tabular}

Table -3 shows the effect of radiation parameter, Prandtl number and time on the Nusselt number (the rate of heat transfer) at the moving and stationary plates. We see that the Nusselt number at the moving plate $N u_{o}$ is increasing with increasing values of radiation parameter and Prandtl number but decreasing with increasing values of time $t$. The Nusselt number at the stationary plate $N u_{1}$ is decreasing with increasing values of radiation parameter and Prandtl number but increasing with increasing values of time $t$.

Table - 4: Sherwood Number

\begin{tabular}{|c|c|}
\hline$S c$ & $S h$ \\
\hline 0.22 & 1.205984 \\
\hline 0.30 & 1.165708 \\
\hline 0.60 & 1.116927 \\
\hline 0.66 & 1.065442 \\
\hline 0.78 & 1.003113 \\
\hline
\end{tabular}


Table - 5: Comparison of Skin - friction $\left(\tau_{o}\right)$ results with the skin - friction $\left(\tau_{o}^{*}\right)$ results of Rajput and Sahu [36] in case of impulsive movement of the plate

\begin{tabular}{|c|c|c|c|c|c|c|}
\hline$G r$ & $\operatorname{Pr}$ & $M$ & $R$ & $t$ & $\tau_{o}$ & $\tau_{o}^{*}$ \\
\hline 5.0 & 0.71 & 2.0 & 1.0 & 0.02 & 3.323170 & 3.323167 \\
\hline 10.0 & 0.71 & 2.0 & 1.0 & 0.02 & 3.813334 & 3.813330 \\
\hline 5.0 & 10.0 & 2.0 & 1.0 & 0.02 & 2.574285 & 2.574278 \\
\hline 5.0 & 0.71 & 4.0 & 1.0 & 0.02 & 3.177202 & 3.177192 \\
\hline 5.0 & 0.71 & 2.0 & 2.0 & 0.02 & 3.304121 & 3.304115 \\
\hline 5.0 & 0.71 & 2.0 & 1.0 & 0.04 & 1.889310 & 1.889301 \\
\hline
\end{tabular}

Table - 6: Comparison of Skin - friction $\left(\tau_{o}\right)$ results with the skin - friction $\left(\tau_{o}^{*}\right)$ results of Rajput and Sahu [36] in case of uniformly accelerated movement of the plate

\begin{tabular}{|c|c|c|c|c|c|c|c|}
\hline$G r$ & $\operatorname{Pr}$ & $M$ & $R$ & $t$ & $F$ & $\tau_{o}$ & $\tau_{o}^{*}$ \\
\hline 5.0 & 0.71 & 2.0 & 1.0 & 0.02 & 0.5 & -0.431103 & -0.431103 \\
\hline 10.0 & 0.71 & 2.0 & 1.0 & 0.02 & 0.5 & -0.340951 & -0.340951 \\
\hline 5.0 & 10.0 & 2.0 & 1.0 & 0.02 & 0.5 & -0.580015 & -0.580015 \\
\hline 5.0 & 0.71 & 4.0 & 1.0 & 0.02 & 0.5 & -0.327830 & -0.327830 \\
\hline 5.0 & 0.71 & 2.0 & 2.0 & 0.02 & 0.5 & -0.450175 & -0.450175 \\
\hline 5.0 & 0.71 & 2.0 & 1.0 & 0.04 & 0.5 & -0.302497 & -0.302497 \\
\hline 5.0 & 0.71 & 2.0 & 1.0 & 0.02 & 0.9 & -0.368107 & -0.368107 \\
\hline
\end{tabular}

Table -4 shows the effect of Schmidt number on the Sherwood number (the rate of mass transfer). From this table, we see that the Sherwood number is decreasing with increasing values of Schmidt number. In order to ascertain the accuracy of the numerical results, the present results are compared with the previous results of Rajput and Sahu[36]in tables $-5,6$ and 7 for both the cases I and II. They are found to be in an excellent agreement.

Table - 7: Comparison of Nusselt number $\left(N u_{o} \& N u_{1}\right)$ results with the Nusselt number $\left(N u_{o}^{*} \& N u_{1}^{*}\right)$ results of Rajput and Sahu [36]

\begin{tabular}{|c|c|c|c|c|c|c|}
\hline $\operatorname{Pr}$ & $R$ & $t$ & $N u_{o}$ & $N u_{o}^{*}$ & $N u_{1}$ & $N u_{1}^{*}$ \\
\hline 0.71 & 1.0 & 0.02 & 2.200655 & 2.200651 & 0.098109 & 0.098098 \\
\hline 10.0 & 1.0 & 0.02 & 2.157629 & 2.157622 & 0.002508 & 0.002487 \\
\hline 0.71 & 2.0 & 0.02 & 2.403843 & 2.403840 & 0.025352 & 0.025335 \\
\hline 0.71 & 1.0 & 0.04 & 2.557641 & 2.557636 & 0.164655 & 0.164624 \\
\hline
\end{tabular}

\section{Conclusions:}

In this present paper, the effects of radiation, heat and mass transfer on an unsteady two - dimensional natural convective Couette flow of a viscous, incompressible, electrically conducting fluid between two parallel plates with suction, embedded in a porous medium, under the influence of a uniform transverse magnetic field. The problem is described by a system of coupled nonlinear partial differential equations, are solved by the finite difference method. A parametric study is performed to illustrate the influence of thermo physical parameters on the velocity, temperature and concentration profiles. It has been shown that: 
1. The fluid velocity increases with increase in $G r, G c, F$ and time $t$.

2. The fluid velocity decreases with increase in $\operatorname{Pr}, S c$ and $R$.

3. The fluid velocity increases with increase in $M$ (in case of impulsive movement of the plate) but it decreases with increase in $M$ (in case of uniformly accelerated movement of the plate).

4. The fluid temperature decreases with increase of $\operatorname{Pr}$ and $R$.

5. The fluid temperature increases with increase of $t$.

6. The fluid concentration decreases with increase of $S c$.

7. The skin - friction increases with increase in $G r, G c, F$ and time $t$.

8. The skin - friction decreases with increase in $\operatorname{Pr}, S c$ and $R$.

9. The skin - friction decreases with increase in $M$ (in case of impulsive movement of the plate) but it increases with increase in the $M$ (in case of uniformly accelerated movement of the plate).

10. The Nusselt number $N u_{o}$ at the moving plate increases with increase in $R$ and $\operatorname{Pr}$ but it decreases with increase in time $t$.

11. The Nusselt number $N u_{1}$ at the moving plate increases with increase in $R$ and $\operatorname{Pr}$ but it decreases with increase in time $t$.

12. In order to ascertain the accuracy of the numerical results, the present results are compared with the previous results of Rajput and Sahu [36]for both the cases I and II. They are found to be in an excellent agreement.

\section{References:}

[1]. Bejan, A., (1993). Convection heat transfer,2nd ed., John Wiley\& Sons.

[2]. Gebhart, B. andPera, L., (1971). The nature of vertical natural convection flows resulting from the combined buoyancy effects of thermal and mass diffusion, Int. J. Heat Mass Transfer,

Vol. 14, pp. 2025- 2050.

[3]. Raptis, A. A., (1982). Free convection and mass transfer effects on the oscillatory flow past an infinite moving vertical isothermal plate with constant suction and heat sources,Astrophy. Space Sci.,Vol. 86, pp. 43-53.

[4]. Bejan, A. andKhair, K. R., (1985). Heat and mass transfer by natural convection in a porous medium, Int. J. Heat and Mass Transfer,Vol. 28, pp. 909-918.

[5]. Jang, J. Y. and Chang, W. J., (1988). Buoyancy - Induced inclined boundary layer flow in a porous medium resulting from combined heat and mass buoyancy effects, Int.Commun. Heat Mass Transfer,Vol. 15, pp. 17-30.

[6]. Lai, F. C. andKulacki, F. A., (1981). Non - Darcy mixed convection along a vertical wall in a saturated porous medium,J. Heat Transfer,Vol. 113, pp. 252-255.

[7]. Nakayama, A. andHossain, M. A., (1995). An integral treatment for combined heat and mass transfer by natural convection in a porous medium, Int. J. Heat and Mass Transfer, Vol. 38, pp. $761-765$.

[8]. Yih, K. A., (1997). The effect of transpiration on coupled heat and mass transfer in mixed convection over a vertical plate embedded in a saturated porous medium, Int.Commun. Heat Mass Transfer,Vol. 24, pp. $265-275$.

[9]. Chamkha, A. J., Takhar, H. S. and Soundalgekar, V. M., (2001). Radiation effects on free convection flow past a semi - infinite vertical plate with mass transfer,Chem. Eng. J., Vol. 84, pp. 335- 342.

[10]. Ganesan, P. and Palani, G.,(2003). Natural convection effects on impulsively started inclined plate with heat and mass transfer, Heat Mass Transfer,Vol. 39, pp. 277-283.

[11]. Eckert, E. R. and Drake, R. M., (1972). Analysis of heat and mass transfer,Mc - Graw Hill.

[12]. Gebhart. B., Jaluria, Y., Mahajan, R. L. and Sammakia, B., (1998).Buoyancy Induced Flow and Transport, New York Hemisphere.

[13]. Nield, D. A. and Bejan, A., (2006). Convection in porous media,3rd ed., Springer.

[14]. Pop, I. and Ingham, D. B., (2001). Convective heat transfer: mathematical and computational modelling of viscous fluids and porous media, OxfordPergamon.

[15]. Incropera, F. P., Bergman, T. L., Lavine, A. S. and Dewitt, D. P., (2011). Fundamentals of heat and mass transfer,John Wiley \& Sons. 
[16]. Bejan, A. and Khair, K. R., (1985). Heat and mass transfer by natural convection in a porous medium, Int. J. Heat Mass Transfer, Vol. 28, pp. $909-918$.

[17]. Raptis, A., Tzivanidis, G.and Kafousias, N., (1981). Free convection and mass transfer flow through a porous medium bounded by an infinite vertical limiting surface with constant suction, Lett. Heat Mass Transfer, Vol. 8, pp. $417-424$.

[18]. Lai, F. C. and Kulacki, F. A., (1991). Coupled heat and mass transfer by natural convection from vertical surfaces in a porous medium, Int. J. Heat Mass Transfer, Vol. 34, pp. $1189-1194$.

[19]. Raptis, A., (1986). Flow through a porous medium in the presence of magnetic field, Int. J. Energy Res., Vol. 10, pp. $97-101$.

[20]. Soundalgekar, V. M., (1990). Free - convection effects on the oscillatory flow past an infinite vertical porous plate with constant suction, J. Roy. Soc. London, Vol. A333, pp. $25-36$.

[21]. Takhar, H. S. and Ram, P. C., (1994). Magnetohydrodynamic free convection flow of water at $4^{\circ} \mathrm{C}$ through a porous medium, Int. Comm. Heat Mass Transfer,Vol.21, pp. 371 - 376.

[22]. Soundalgekar, V.M., Gupta, S.K. and Birajdar, N.S., (1979). Effects of masstransfer and free convection effects on MHD Stokes problem for avertical plate, Nucl.Eng.Design,Vol.53, pp. $309-346$.

[23]. Eilbashbeshy, E.M.A.,(1997). Heat and Mass transfer along a verticalplate with variable surface tension and concentration in the presenceof magnetic field,Int.J.Eng Science,Vol. 35, pp. $515-522$.

[24]. Alagoa, K.D., Tay, G. and Abbey, T.M.,(1999). Radiative and freeconvection effects of a MHD flow through porous medium betweeninfinite parallel plates with time dependent suction, Astrophysics.Space. Sci.,Vol.260, pp. $455-468$.

[25]. Bestman, A.R. and Adjepong, S.K.(1998). Unsteady hydromagneticfree convection flow with radiative transfer in a rotating fluid, Astrophysics. Space Sci.,Vol.143, pp.73- 80 .

[26]. Promise Mebine and Emmanuel MunakuroghaAdigio, (2011). Effects of thermal radiation on transient MHD free convection flow over a vertical surface embedded in a porous medium with periodic boundary temperature, MathematicaAeterna, Vol. 1, No. 4, pp. $245-261$.

[27]. Ramachandra Prasad, V. Bhaskar Reddy, N., (2007). Radiation and mass transfer effects on an unsteady MHD free convection flow past a heated vertical plate in a porous medium with viscous dissipation, Theoret. Appl. Mech., Vol. 34, No. 2, pp. $135-160$.

[28]. Israel - Cookey, C., Ogulu, A. and Omubo - Pepple. (2003). Influence of viscous dissipation on unsteady MHD free convection flow past an infinite vertical plate in porous medium with time dependent suction, Int. J. Heat and Mass Transfer, Vol. 46, pp. $2305-2311$.

[29]. Singh, A. K., (1988). Natural convection in unsteady Couette motion, Defence Science Journal, Vol. 38, No. 1, pp. $35-41$.

[30]. Kearsley, A. J., (1994). A steady state model of Couette flow with viscous heating, Int. J. Eng. Tech. Research, Vol. 32, pp. $179-186$.

[31]. Jha, B. K., (2001). Natural convection in unsteady MHD Couette flow, Heat and mass transfer, Vol. 37, pp. 329 331.

[32]. Mebine, P., (2007). Radiation effects on MHD Couette flow with heat transfer between two parallel plates, Global J. Pure applied Mathematics, Vol. 3, No. 2, pp. $191-202$.

[33]. Narahari, M., (2010). Effects of thermal radiation and free convection currents on the unsteady Couette flow between two vertical parallel plates with constant heat flux at one boundary, Wseas Transactions on heat and mass transfer, Vol. 5, pp. $21-30$.

[34]. Seth, G. S., Ansari, M. S. and Nandkeolyar (2011). Unsteady hydromagneticCouette flow within a porous channel, Tamkang J. Sci. Eng., Vol. 14, No. 1, pp. 7 - 14.

[35]. Deka, R. K. and Bhattacharya, A., (2011). Unsteady free convective Couette flow of heat generation/absorbing fluid in porous medium, Int. J. Math. Archive, Vol. 2, No. 6, pp. $853-863$.

[36]. Rajput, U. S. and Sahu, P. K., (2012). Natural convection in unsteady hydromagneticCouette flow through a vertical channel in the presence of thermal radiation, Int. J. of Appl. Math. and Mech.,Vol. 8, No. 3, pp. 35 - 56.

[37]. Brewster, M. Q., (1992). Thermal radiative transfer \& properties, John Wiley \& Sons.

[38]. Antia, M., (1991). Numerical Methods for Scientists and Engineers, Tata McGraw - Hill, New Delhi. 